\title{
Design and Optimization of Fresnel Lens for High Concentration Photovoltaic System
}

\author{
Lei Jing, Hua Liu, Yao Wang, Wenbin Xu, Hongxin Zhang, and Zhenwu Lu
}

Opto-Electronic Technology Center, Changchun Institute of Optics, Fine Mechanics and Physics, Chinese Academy of Sciences, Changchun 130033, China

Correspondence should be addressed to Hua Liu; 54153023@qq.com

Received 27 December 2013; Revised 22 February 2014; Accepted 24 February 2014; Published 30 March 2014

Academic Editor: Dimitrios Karamanis

Copyright (C) 2014 Lei Jing et al. This is an open access article distributed under the Creative Commons Attribution License, which permits unrestricted use, distribution, and reproduction in any medium, provided the original work is properly cited.

A practical optimization design is proposed, in which the solar direct light spectrum and multijunction cell response range are taken into account in combination, particularly for the Fresnel concentrators with a high concentration and a small aspect ratio. In addition, the change of refractive index due to temperature variation in outdoor operation conditions is also considered in the design stage. The calculation results show that this novel Fresnel lens achieves an enhancement of energy efficiency of about $10 \%$ compared with conventional Fresnel lens for a given solar spectrum, solar cell response, and corrected sunshine hours of different ambient temperature intervals.

\section{Introduction}

Because of the expensive cost and quality degradation, high reflectivity mirrors are not recommended for a concentration photovoltaic system (CPVs) [1], and optical refractive polymer components such as Fresnel lenses are most frequently used for commercial CPVs, which possess advantages of compactness, simplicity in manufacture, and relative cheapness [2-4]. However, the Fresnel lenses also have several inherent disadvantages.

The first is the dispersion of the solar spectrum, which leads to an essential decrease of the energy efficiency of the solar cells, especially for the Fresnel lens having a high concentration and a small aspect ratio (i.e., the ratio of focal length to the aperture of lens). The previous Fresnel lens design is mainly based on the concept that all the grooves are with the same design wavelength $\lambda$, which is popularly chosen to be either $0.54 \mu \mathrm{m}$ [5] or the center wavelength of the solar spectrum limit [6], respectively, for the consideration of the relative peak irradiance of solar radiation and the balance of dispersion effect. Then each groove profile is obtained at this wavelength by means of simple trigonometry. These investigations were mostly devoted to analyzing the effect of dispersion of solar spectrum of the solar cell after designing, rather than to considering the solar direct light spectrum in the design stage of Fresnel lens [6-10]. Although Watson and Jayroe Jr. [11] presented a method for the design of Fresnel lens with consideration of solar spectrum, the multijunction cell response and the influence of ambient temperature variation were not considered in design process.

The second is the variation of outdoor temperature conditions during operation, which directly affects the spectral decomposing of the Fresnel lens. Usually Fresnel lens concentrator modules are characterized under standard testing conditions by subjecting them to an irradiation of $1000 \mathrm{~W} / \mathrm{m}^{2}$ with an AM1.5D spectrum and a temperature of $20^{\circ} \mathrm{C}$ [12]. Nevertheless, the modules would not perform in accordance under actual conditions. Their efficiency is strongly affected by the environmental fluctuations. The previous studies paid more attention to the analysis of optical performances of CPVs affected by the variation of temperatures outdoors [13, 14]. The temperature dependence of refractive index caused thermal effects which had rarely been taken into account in the design stage of Fresnel lens.

With this background, in this paper, a more realistic optimization method is proposed to design a high concentration Fresnel lens with a small aspect ratio. Each groove is assumed with a specific design main wavelength related to 


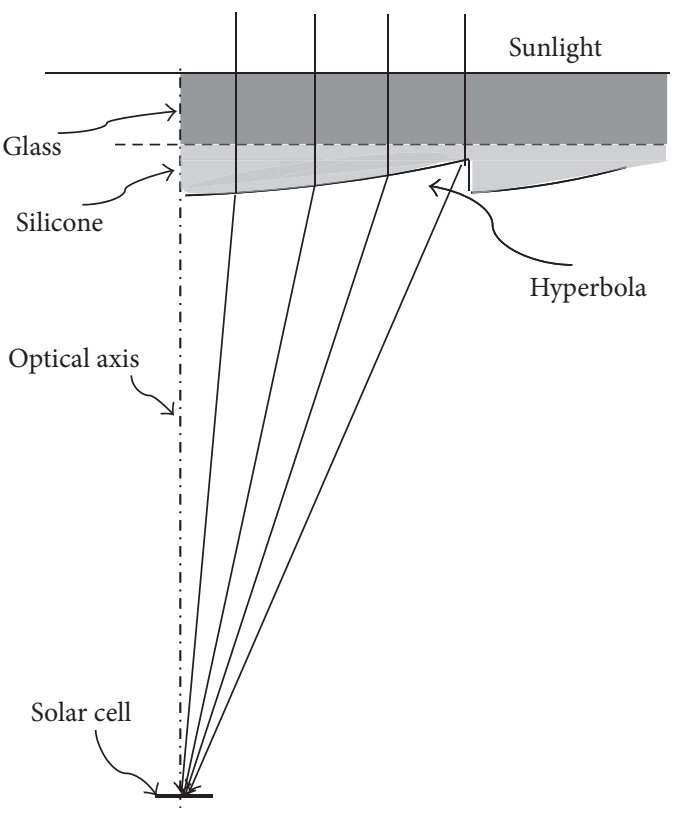

FIGURE 1: Determination of the profile for each groove with the main wavelength $\lambda$. Only one center groove of Fresnel lens is drawn for interpreting.

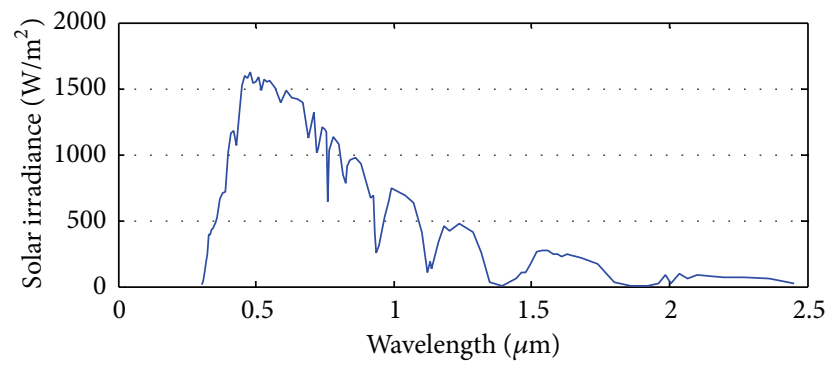

FIGURE 2: The irradiance distribution characteristic of AM1.5D solar spectrum.

the solar spectrum and the solar cell response. Then golden section search method is used to search the optimal design main wavelength to obtain the maximal energy efficiency of the solar cell. In addition, the influence of the change of refractive index caused by ambient temperature variation is also considered in the design process.

\section{Optimization Design Method}

For simplicity (in both designing and manufacturing), we restrict ourselves to the case as follows. The Fresnel lens array is replicated in one piece into a silicone rubber on glass; the exit surface of each groove is curvilinear and the depth of each groove is identical. As shown in Figure 1, the exit surface profile of each groove can be easily determined as a portion of a hyperbola in the condition that the parallel sunlight beam with a design wavelength $\lambda$ is focused on the center of solar cell [15].
Considering the excellent performance of the solar cell at high concentration sunlight, one of the purposes of our work is to design a nonimaging Fresnel lens used in CPVs with a high concentration factor and an aspect ratio of a relatively small value.

For the most efficient design of Fresnel lens for CPVs, the lens should focus maximum amount of energy in a small spot size [1]. For this, an appropriate design method of the Fresnel lens which guarantees the CPVs with maximal energy collection efficiency is needed. In the next section we will describe the realistic optimization design method in detail, with the consideration of the solar spectrum, the multijunction cell response, and the variation of the ambient temperatures.

2.1. Consideration of the Solar Spectrum. For Fresnel lens concentrators, the solar radiation spectrum mostly used in the analysis is in the range of AM1.5D (at sea level) [16]. As can be seen in Figure 2, it has a quite wide wavelength range of 300-2500 $\mathrm{nm}$. Considering the multijunction solar cell response, we are only interested in wavelength range of $350-1750 \mathrm{~nm}$ [17]. However, this spectrum range is still too wide for the Fresnel lens with serious dispersion effect. If we force all the wavelengths of sunlight to be collected by solar cell for a given relatively small aspect ratio, such as that often used in commercial CPVs of 1.2, the dimension of solar cell would be rather larger, corresponding to a small concentration factor of $175 \mathrm{X}$ (spectrum of $350-1750 \mathrm{~nm}$ ). In other words, in order to achieve a high concentration, the dimension of solar cell should not be too large, and all the sunlight would be not captured by the relatively small solar cell (as shown in Figure 3).

Figure 3 illustrates schematically how the sunlight beam passes through an arbitrary groove of Fresnel lens and is intercepted by a small solar cell. It is noted that the dimensions of the solar cell and spectrum are locally exaggerated for the interpreting conveniently. The sunlight is dispersed at the focal plane of the Fresnel lens due to the wavelength dependence of refractive index of lens material $n=n(\lambda)$ and then is intercepted by the relatively small solar cell. The blue and red zones denote the loss of shorter and longer wavelength beam, respectively. Thus, the solar radiation spectrum interval effective for the solar cell changes from the entire spectrum $\left(\lambda_{S}, \lambda_{L}\right)$ to the intercepted spectrum $\left(\lambda_{a}, \lambda_{b}\right)$.

For each groove, any wavelength can be designated as the main wavelength of lens design. Since the incident flux of solar spectrum is wavelength dependent (on its way through the atmosphere, the sunlight suffers from Rayleigh scattering, aerosol extinction, and absorption of water vapor, ozone, and nitrogen dioxide), different design wavelength results in variety of the spectrum intervals corresponding to the change of captured energy. The energy can be expressed by integration:

$$
E_{\text {groove }}=\int_{\lambda_{a}}^{\lambda_{b}} I_{\mathrm{ns}} * T_{\text {ave }} d \lambda,
$$

where $E_{\text {groove }}$ denotes the collected energy of the solar cell for an arbitrary groove, $T_{\text {ave }}$ denotes the average transmission 


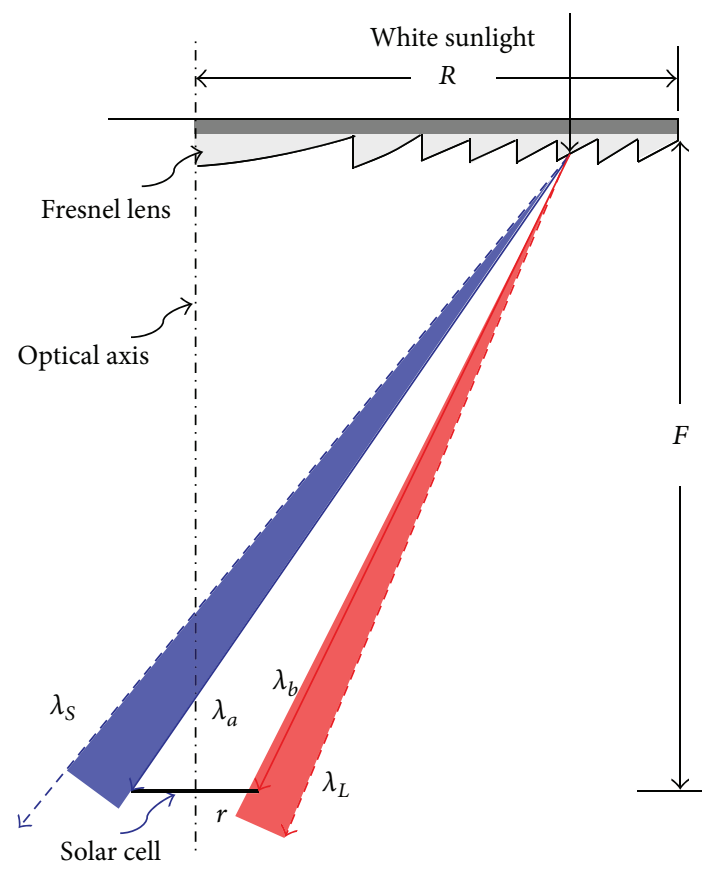

FIGURE 3: Plot of sunlight dispersion through an arbitrary groove of a Fresnel lens with aspect ratio 1.2, geometrical concentration 1200X, and design spectrum $350-1750 \mathrm{~nm}$. It is noted that the dimensions of cell and spectrum are locally exaggerated.

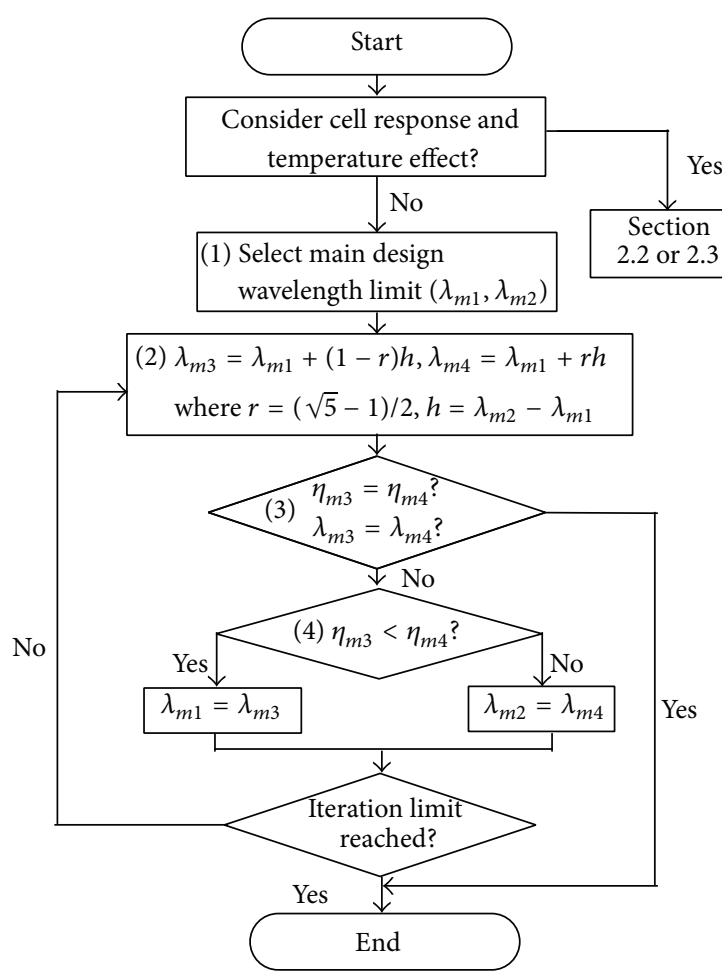

FIgURE 4: Flow chart of golden section search method for calculation of the main design wavelength. factor, and $I_{\text {ns }}$ denotes the normalized irradiance of solar radiation. For a given solar cell radius of $r$ with Fresnel lens semidimension of $R$, focus of $F$, and depth of groove of $d$, both the intercepted spectrum interval and corresponding collected energy can be calculated using simple trigonometry for each groove $[2,11]$.

In view of the solar radiation spectrum normalization and its dependence on specified geographical location, it is more appropriate to use the collected energy efficiency $\eta_{n}$ to evaluate the final performance of each groove, which is the ratio of energy collected by solar cell to solar radiation energy:

$$
\eta_{n}=\frac{E_{\text {groove }}}{E_{\text {total }}}=\frac{\int_{\lambda_{a}}^{\lambda_{b}} I_{\mathrm{ns}} \cdot T_{\mathrm{ave}} d \lambda}{\int_{\lambda_{S}}^{\lambda_{L}} I_{\mathrm{ns}} \cdot T_{\mathrm{ave}} d \lambda} .
$$

The total energy efficiency collected by the solar cell can be written as

$$
\eta_{\text {total }}=\sum_{n}^{N} \frac{\eta_{n} \cdot S_{n}}{S}, \quad n=1,2,3, \ldots, N,
$$

where $S_{n}$ and $S$ are the area of each groove and the total incident area of Fresnel lens, respectively, and $N$ is the number of the grooves.

The objective of optimization design is to find the optimal main wavelength of design, corresponding to the maximal energy efficiency, which can be potentially realized via an iterative search, such as the well-known golden section search method. The flow chart of the basic procedure is shown in Figure 4 and the major steps of the algorithm are listed as follows. At first we determine whether we take into account the solar cell response and temperature effects. If yes, see Sections 2.2 or 2.3; otherwise, the following steps begin. (1) Select the main design wavelength limit $\left(\lambda_{m 1}, \lambda_{m 2}\right)$. (2) Calculate the golden section points of $\lambda_{m 3}$ and $\lambda_{m 4}$. (3) Determine whether the utilized energy efficiency $\eta_{m 3}$ equals $\eta_{m 4}$ and whether $\lambda_{m 3}$ equals $\lambda_{m 4}$. If the condition is satisfied, the iteration finishes. (4) Compare the solar cell efficiency $\eta_{m 3}$ with $\eta_{m 4}$. If $\eta_{m 3}$ is smaller than $\eta_{m 4}$, make $\lambda_{m 1}=\lambda_{m 3}$. Otherwise, $\lambda_{m 2}=\lambda_{m 4}$. Then, judge whether the iteration limit reaches, if it reaches, the iteration finishes. If not, come back to step (2) and continue iteration. In general, the optical performance of the Fresnel lens is automatically improved by the iteration, and the iteration converges to a good solution at final.

2.2. Consideration of the Solar Cell Response. III-V based multijunction cells can operate easily at very high concentration levels and so they are excellent for high concentration system. For example, metamorphic monolithic dual-junction cells of GaInp/GaInAs achieved an efficiency of 30\% at 1000 suns. A monolithic triple-junction cell of GaInP/GaInAs/Ge achieved efficiency of $39 \%$ at a concentration of 236 suns [18]. Multijunction solar cells consist of a monolithic stack of semiconductor structures of different materials. Each material has a different band-gap, and the junction is with a certain conversion range of the solar spectrum $[19,20]$ (Figure 5). Therefore, the combination of solar direct light 


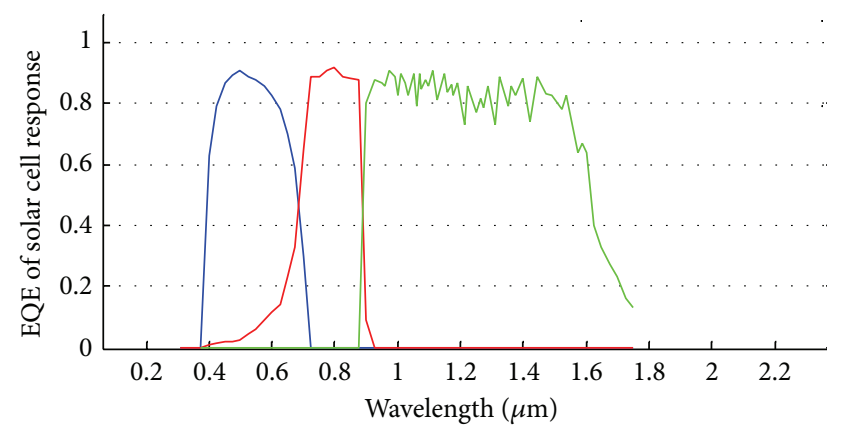

EQE of top junction
EQE of middle junction
EQE of bottom junction

Figure 5: The different external quantum efficiency of triplejunction solar cell.

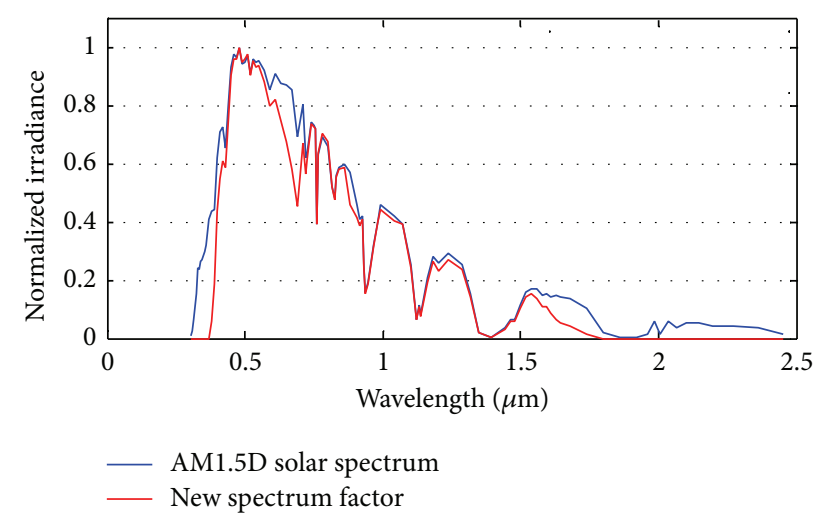

Figure 6: The introduced spectrum factor for consideration of the solar cell response.

spectrum and cell response range should be taken into account in the design stage of Fresnel lens.

In order to make the design more convenient, a spectrum factor of $I_{\mathrm{sc}}$ is introduced in this paper, which is defined as the product of the normalized irradiance of solar radiation $I_{\text {ns }}$ (it is dependent on geographical location where the CPVs is actually installed) and the external quantum efficiency of solar cell $\eta_{\mathrm{EQE}}$ (Figure 6):

$$
I_{\mathrm{sc}}=I_{\mathrm{ns}} \cdot \eta_{\mathrm{EQE}} \cdot
$$

The characteristic of the normalized irradiance distribution of the spectrum factor that describes the compound relationship of the solar spectrum and the solar cell response will be specified as the design spectrum. In further design, the spectrum factor will substitute the solar spectrum in the optimization process mentioned above.

2.3. Consideration of the Outdoor Ambient Temperature. CPVs technology is particularly effective in areas of high direct normal incident (DNI) radiation (sunny locations), such as desert or high mountain plateaus. However, the climate there is often much harsher than the standard indoor

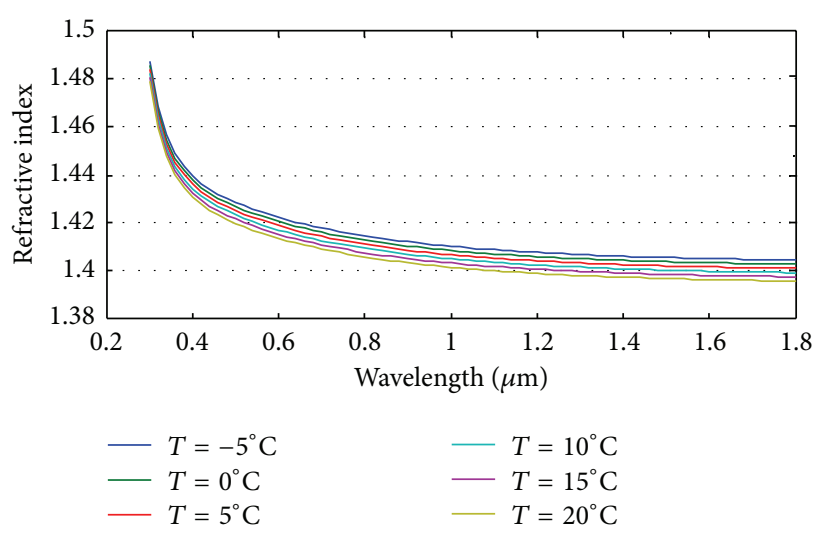

Figure 7: The dependence of refractive index of silicone on temperature: $K=-3.4 e-4^{\circ} \mathrm{C}^{-1}$.

conditions. For example, the ambient temperatures of CPV may go below freezing point in winter and up to more than $40^{\circ} \mathrm{C}$ in summer in desert. It deviates significantly from the conventional designing and testing standard temperature of $20^{\circ} \mathrm{C}$, which leads to serious power degradation. The temperature dependence of refractive index of concentrator material may account for the degradation, especially for the polymer material of Fresnel lens [21]. The variation of the refractive index causes a shift of the focal plane away from or close to the Fresnel lens as temperature rises or falls. Thus, the temperature dependence of the refractive index of Fresnel lens material is also considered in the design of the research. Further analysis of other effects of temperature variation will be investigated in next research. A number of studies have been devoted to direct measurement of specific volume variations with respect to temperature for polymers [22]. For instance, the temperature dependence of the refractive index of silicone lens material is almost independent of wavelength and can be approximated by a linear relationship [23] very well in the interest range (Figure 7):

$$
n=n_{0}+k \cdot \Delta T
$$

where $n_{0}$ is the original refractive index, $k$ is the temperature coefficient, and $\Delta T$ is the difference of temperature.

In this paper, for simplicity, we only consider the outdoor temperature variation during one day to optimize the Fresnel lens to achieve maximal energy efficiency. Having the monthly or annual solar radiation and temperature data adequately, the optimal design main wavelength for each groove and the maximal total energy efficiency could be obtained in the same way. Since the CPVs is usually exposed in outdoor environments for a long time, the operative temperature of Fresnel lens is assumed to be consistent with the ambient temperature. As shown in Figure 7, the temperature directly affects the refractive index of Fresnel lens material and finally affects the energy efficiency of Fresnel lens. Therefore, the optimization procedure may consider sunshine hours of 


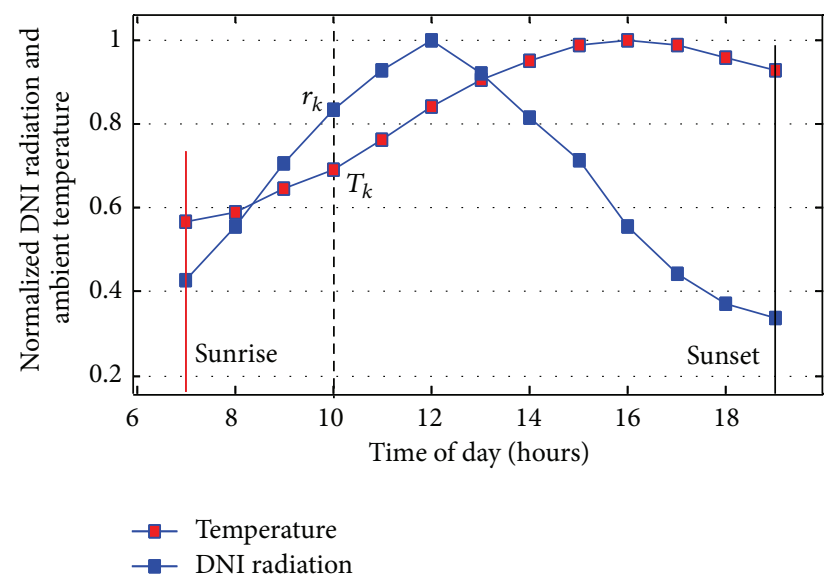

FIGURE 8: The normalized diurnal solar DNI radiation and ambient temperature.

different temperature intervals, and then the evaluation index $\eta$ of the optimization can be rewritten as

$$
\eta_{T}=\frac{\sum_{m=1}^{M} \eta_{T_{m}} \cdot H_{T_{m}}}{\sum_{m=1}^{M} H_{T_{m}}}
$$

where $\eta_{T_{m}}$ represents the energy efficiency for solar cell with ambient temperature $T_{m}, H_{T_{m}}$ represents the corresponding normalized diurnal amount of sunshine hours, and $M$ represents the number of temperature intervals.

Generally speaking, the change of ambient temperature is primarily determined by the variation of solar radiation. Different ambient temperature always relates with different solar DNI radiation. Consequently, in the optimization process, the solar DNI radiation factor with different ambient temperature needs to be also included. As shown in Figure 8, in a clear and sunny location, though the normal diurnal variation of ambient temperatures is not simply dependent on solar DNI radiation and there is a slight delay in the response of temperature to changes in the solar DNI radiation, we do not pay too much attention to the specific formula of the solar DNI radiation and the ambient temperature. We only need to obtain the solar DNI radiation value of $r_{k}$ at the ambient temperature value of $T_{k}$ (Figure 8) to enhance the effect of the temperature. Thus, the normalized diurnal sunshine hours should be further corrected by the solar DNI radiation as

$$
H_{T_{m}}=\frac{\sum_{k=1}^{L} r_{k}}{\sum_{k=1}^{K} r_{k}}, \quad 1<L<K,
$$

where $r_{k}$ is the relevant solar DNI radiation at the temperature $T_{k}, K$ is the total diurnal sunshine hours in one day, and $L$ is the sunshine hours at the identical temperature intervals.

With the same optimization method as the optimization of solar spectrum mentioned above, as well as (6) and (7), the optimal design main wavelength for each groove and total maximal energy efficiency could be finally calculated under the consideration of ambient varying temperatures in one day.

\section{Example and Discussion}

A simple high concentration circular Fresnel lens is designed and simulated to validate this novel design method. The specifications of the design parameters are as follows. The diameter and the focal length of Fresnel lens are, respectively, $270 \mathrm{~mm}$ and $320 \mathrm{~mm}$, the diameter of solar cell is $7.78 \mathrm{~mm}$, and the solar half angle is $0.27^{\circ}[24,25]$. The geometrical concentration is $1200 \mathrm{X}$ and the aspect ratio is 1.2. For this study we choose polymeric plastic silicone as the base material of Fresnel lens. This is a very good option with high transmission factor, susceptible of molding manufacture and low cost. Its temperature coefficient is $k=-3.4 e-4^{\circ} \mathrm{C}^{-1}$. The depth of each groove has an identical value of $0.5 \mathrm{~mm}$, which can be freely adjusted under manufacturing condition. The characteristic of multijunction cell response is shown in Figure 5. The geographical location of CPVs installed practically is selected at west of China, Qinghai-Tibet Plateau. It is a good location for constructing the solar power plant due to its greatly abundant solar resource with the annual radiation reaching the ground mostly more than $6700 \mathrm{MJm}^{-2}$ year $^{-1}$ (the data stems from China Meteorological Administration). More importantly, building power plant at this place will solve the problem of power shortage there and improve the living standard and the life quality of the western people.

Figure 9 shows the variation of solar DNI radiation as the ambient temperature in mid-June of Waliguan in Qinghai, which lies more than 3800 meters and has less cloud in sky. After sunrise solar DNI radiation reaches the ground, and the ambient temperature gradually increases. The solar DNI radiation reaches its maximum around noon time, but the maximum of temperature occurs at 4 p.m. The difference between the diurnal highest and lowest temperature is up to $15^{\circ} \mathrm{C}$ (Figure 9(a)). The maximal daily sunshine hours corrected by DNI radiation occur at temperature of $10^{\circ} \mathrm{C}-$ $15^{\circ} \mathrm{C}$, which is considerably deviated from the conventional designing and testing standard temperature of $20^{\circ} \mathrm{C}$ for Fresnel lens (Figure 9(b)). It is clear that the change of the ambient temperature may badly impact the efficiency utilized by the solar cell.

According to the optimization method described above, the optimal design main wavelength and maximal energy efficiency of solar cell for each groove are finally obtained (Figures 10 and 11).

As shown in Figure 10, the design main wavelength is different for each groove, so that the energy collected by solar cell or the collection efficiency of each groove is bigger than that with the conventional design (Figure 11). It is more distinct particularly for the marginal grooves of the Fresnel lens. This could be attributed to the fact that the closer to the edge of Fresnel lens the grooves are, the more serious the dispersion of spectrum is.

As shown in Table 1, for the conventional designs with main wavelength of $\lambda_{m}=0.54 \mu \mathrm{m}$ and $\lambda_{m}=1.05 \mu \mathrm{m}$, they both have energy efficiency more than $92 \%$. Due to the effect of dispersion of solar spectrum, the energy efficiency reduces to less than $65 \%$. Moreover, due to the effect of the ambient temperature, it further reduces to about $62 \%$ further. But the novel design with the consideration of the solar spectrum 


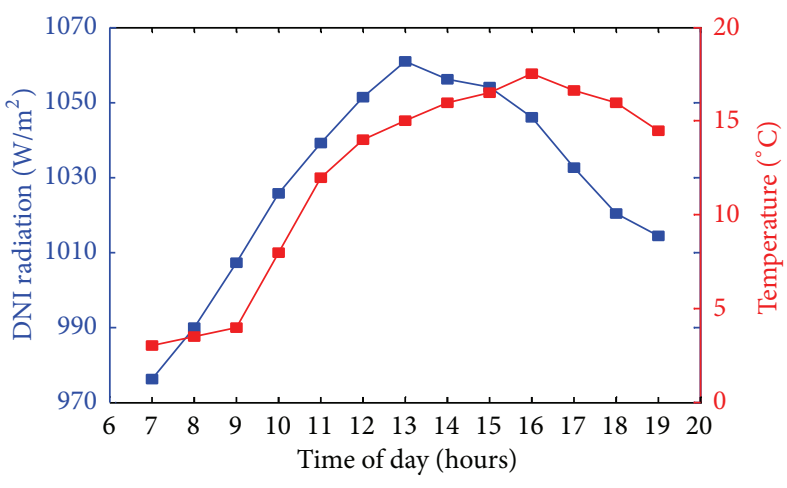

(a)

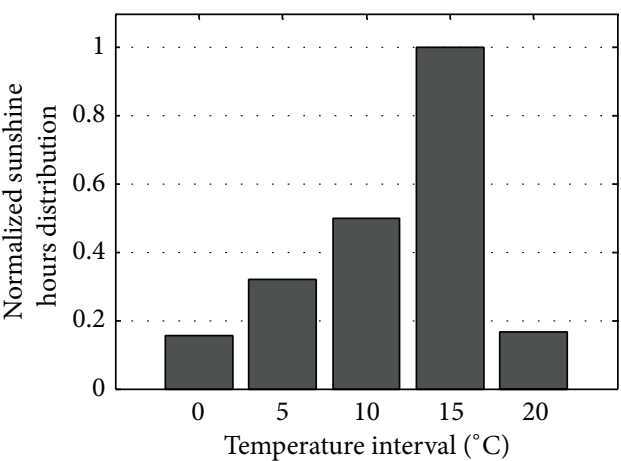

(b)

FIgURE 9: (a) The variation of solar DNI radiation as ambient temperature. (b) The corrected sunshine hours distribution of temperature interval 13th of June in 2006 (Waliguan in Qinghai).

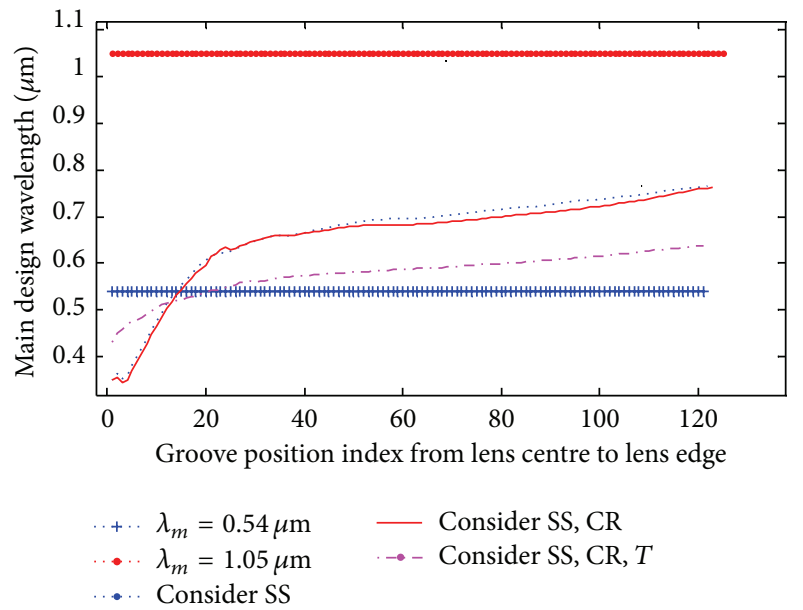

FIGURE 10: The different design main wavelength of each groove for the conventional design and the novel design (SS: solar spectrum; CR: cell response; $T$ : temperature).

can obtain energy efficiency about $73 \%$, which is, respectively, 9.1\% and $9.7 \%$ higher than the conventional design with a constant design main wavelength of $\lambda_{m}=0.54 \mu \mathrm{m}$ and $\lambda_{m}=1.05 \mu \mathrm{m}$, respectively. Once the multijunction cell response and the temperature effects are also in consideration in design, the total efficiency would further increase more than $1.5 \%$.

\section{Conclusions}

A novel optimization design of Fresnel lens used in CPVs was proposed in this paper, in which the solar direct light spectrum, the cell response range, and the change of refractive index resulting from ambient temperature are all taken into account. We assume that each groove of the Fresnel lens has a different design main wavelength and use the golden section method to search the optimal design main wavelength. In this way the intercepted wavelength interval corresponds to the maximal energy efficiency utilized by the solar cell. It is

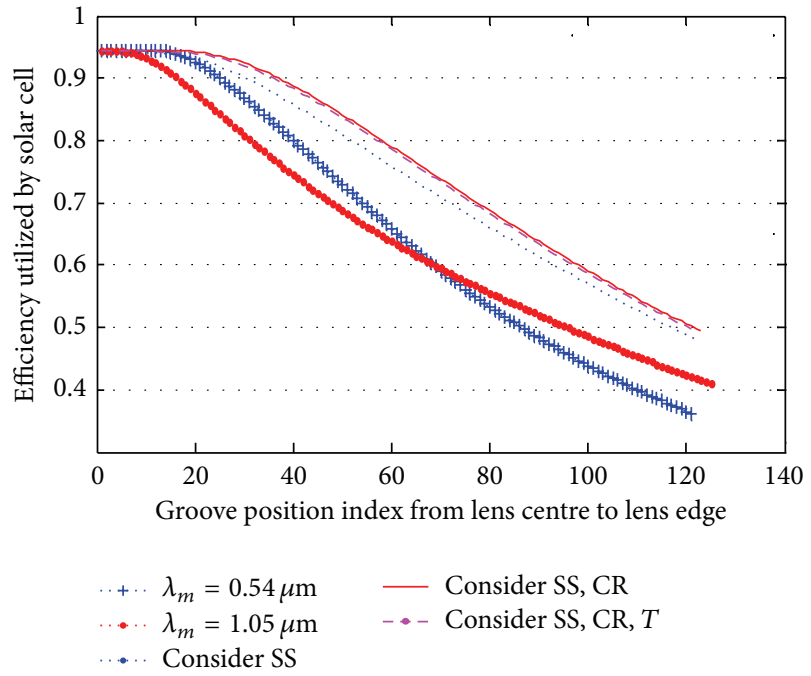

FIGURE 11: The energy efficiency of each groove for the conventional design and the novel design (SS: solar spectrum; CR: cell response; $T$ : temperature).

TABLE 1: The calculated energy efficiency of the conventional design and the novel design for entire Fresnel lens.

\begin{tabular}{lccc}
\hline Design method & \multicolumn{1}{c}{ Efficiency } & Effect of temperature \\
\hline Conventional method & & \\
$\lambda_{m}=0.54 \mu \mathrm{m}$ & SWS $^{\mathrm{d}}$ & $92.4 \%$ & $62.5 \%(\downarrow)$ \\
& MWS $^{\mathrm{e}}$ & $64.73 \%(\downarrow)$ & \\
$\lambda_{m}=1.05 \mu \mathrm{m}$ & SWS & $92.7 \%$ & $62.14 \%(\downarrow)$ \\
New method & MWS & $64.08 \%(\downarrow)$ & \\
Consider SS & & & - \\
Consider SS, CR & $73.84 \%(\uparrow)$ & - \\
Consider SS, CR, $T^{\mathrm{c}}$ & $75.76 \%(\uparrow)$ & - \\
\hline
\end{tabular}

${ }^{\mathrm{a}}$ Solar spectrum; ${ }^{\mathrm{b}}$ cell response; ${ }^{\mathrm{c}}$ temperature; ${ }^{\mathrm{d}}$ single wavelength; ${ }^{\mathrm{e}}$ multiple wavelengths. 
indicated in a comparison of the novel with the conventional design of the Fresnel lens that an increase of energy efficiency more than $9 \%$ can be achieved. These will be helpful to substantially improve the performance of CPVs and reduce its related cost in mass installation. The concentration module based on this novel Fresnel lens is a promising option for the development of a cost-effective photovoltaic solar energy generation.

\section{Conflict of Interests}

The authors declare that there is no conflict of interests regarding the publication of this paper.

\section{Acknowledgments}

The authors sincerely thank Professor Wang ZhaoQi for his help with English language. And the authors gratefully acknowledge the financial support from National Basic Research Program of China under Grant no. 2013AA03A116 and Projects of Science and Technology Development Plan of Jilin Province under Grant no. 20130206018GX.

\section{References}

[1] J. L. Alvarez, V. Díaz, and J. Alonso, "Optics design key points for high gain photovoltaic solar energy concentrators," in Optical Design and Engineering II, vol. 5962 of Proceedings of SPIE, September 2005.

[2] R. Leutz and A. Suzuki, Nonimaging Fresnel Lenses: Design and Performance of Solar Concentrators, Springer Series in Optical Sciences, Springer, 2001.

[3] G. Zubi, J. L. Bernal-Agustín, and G. V. Fracastoro, "High concentration photovoltaic systems applying III-V cells," Renewable and Sustainable Energy Reviews, vol. 13, no. 9, pp. 2645-2652, 2009.

[4] D. Vázquez-Moliní, A. Á. Fernándex-Balbuena, E. Bernabeu et al., "New concentrator multifocal Fresnel lens for improved uniformity design and characterization," in High and Low Concentrator Systems for Solar Electric Applications IV , vol. 7407 of Proceedings of SPIE, 2009.

[5] J. H. Song, J. H. Yu, J. H. Lee et al., "Design of linear Fresnel lens for concentrated photovoltaic system," Advanced Materials Research, vol. 860-863, pp. 32-36, 2014.

[6] E. Lorenzo, "Chromatic aberration effect on solar energy systems using Fresnel lenses," Applied Optics, vol. 20, no. 21, pp. 3729-3732, 1981.

[7] E. Lorenzo and A. Luque, "Fresnel lens analysis for solar energy applications," Applied Optics, vol. 20, no. 17, pp. 2941-2945, 1981.

[8] E. V. Bobkova, V. A. Grilikhes, A. A. Soluyanov, and M. Z. Shvarts, "Effect of chromatic aberration on the concentration of solar radiation by Fresnel lenses," Technical Physics Letters, vol. 32, no. 12, pp. 1039-1042, 2006.

[9] D. Chemisana, A. Vossier, L. Pujol, A. Perona, and A. Dollet, "Characterization of Fresnel lens optical performances using an opal diffuser," Energy Conversion and Management, vol. 52, no. 1, pp. 658-663, 2011.

[10] D. C. Miller, M. D. Kempe, C. E. Kennedy, and S. R. Kurtz, "Analysis of transmitted optical spectrum enabling accelerated testing of multijunction concentrating photovoltaic designs," Optical Engineering, vol. 50, no. 1, Article ID 013003, 2011.

[11] M. D. Watson and R. R. Jayroe Jr., "Fresnel lens solar concentrator design based on geometric optics and blackbody radiation equation," in Nonimaging Optics: Maximum Efficiency Light Transfer V, vol. 3781 of Proceedings of SPIE, pp. 85-93, July 1999.

[12] M. Z. Shvarts, V. M. Andreev, V. S. Gorohov et al., "Flatplate Fresnel lenses with improved concentrating capabilities: designing, manufacturing and testing," in Proceeding of IEEE Photovoltaic Specialists Conference, 2008.

[13] T. Hornung, A. Bachmaier, P. Nitz, and A. Gombert, “Temperature and wavelength dependent measurement and simulation of Fresnel lenses for concentrating photovoltaics," in Photonics for Solar Energy Systems III, vol. 7725 of Proceedings of SPIE, April 2010.

[14] Y.-C. Chen and C.-H. Su, "Integrated opto-mechanical analysis of a PMMA Fresnel lens for a concentrated photovoltaic system," Microsystem Technologies, vol. 19, no. 11, pp. 1725-1729, 2013.

[15] R. Winston, J. C. Miñano, and P. Benítez, Nonimaging Optics, Elsevier-Academic Press, New York, NY, USA, 2005.

[16] ASTM, "Standard tables for reference solar spectral irradiances: direct normal and hemi-spherical on $0.37^{\circ}$ titled surface," ASTM G173-03e1, Book of standards, 2003.

[17] "C1MJ-CDO-100-IC product data sheet," http://www.spectrolab.com.

[18] A. W. Bett, B. Burger, F. Dimroth, G. Siefer, and H. Lerchenmüller, "High-concentration PV using III-V solar cells," in Proceedings of the 4th IEEE World Conference on Photovoltaic Energy Conversion (WCPEC '04), pp. 615-620, May 2006.

[19] N. H. Karam, R. R. King, C. Fetzer et al., "Development and characterization of high-efficiency GaInPGaAsGe dual-and triple-junction solar cells," Proceeding of IEEE Transactions on Electron Devices, vol. 46, no. 10, 1999.

[20] A. L. Luque and V. M. Andreev, Concentrator Photovoltaics, Springer, 2007.

[21] T. Schult, M. Neubauer, Y. Bessler et al., "Temperature dependence of Fresnel lenses for concentrating photovoltaics," in Proceedings of the International CPV Workshop, Darmstadt, Germany, 2009.

[22] P. Michel, J. Dugas, J. M. Cariou, and L. Martin, "Refractiveindex variations with temperature of PMMA and polycarbonate," Journal of Macromolecular Science-Physics, vol. 25, no. 4, pp. 379-394, 1986.

[23] A. Norris, J. DeGroot Jr., F. Nishida, U. Pernisz, N. Kushibiki, and T. Ogawa, "Silicone polymers for optical films and devices," in Linear and Nonlinear Optics of Organic Materials II, vol. 4798 of Proceedings of SPIE, pp. 79-86, July 2002.

[24] L. Reed, R. Winston, and A. Ritschel, "Field results of a Kohler integrating photovoltaic system," in Nonimaging Optics and Efficient Illumination Systems IV, R. Winston and R. J. Koshel, Eds., vol. 6670 of Proceedings of SPIE, August 2007.

[25] J. A. Duffie and W. A. Beckman, Solar Engineering of Thermal Processes, Wiley Interscience, New York, NY, USA, 1980. 

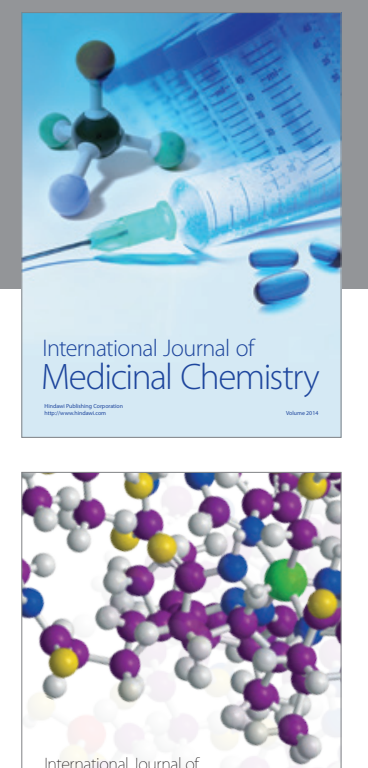

\section{Carbohydrate} Chemistry

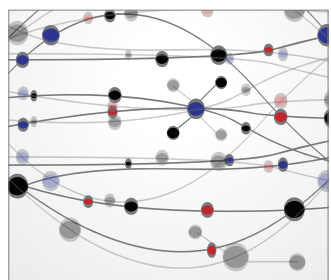

The Scientific World Journal
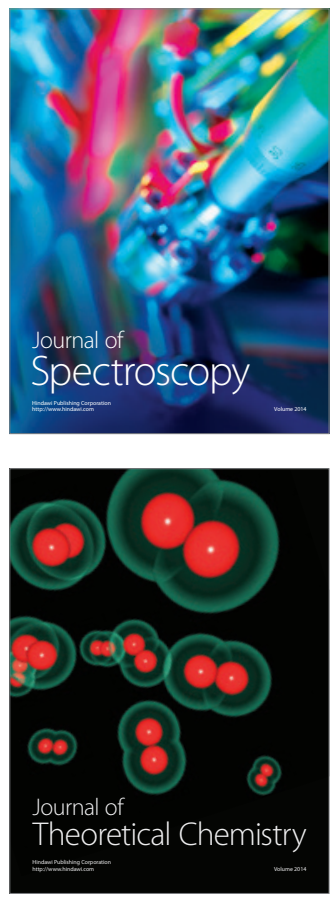
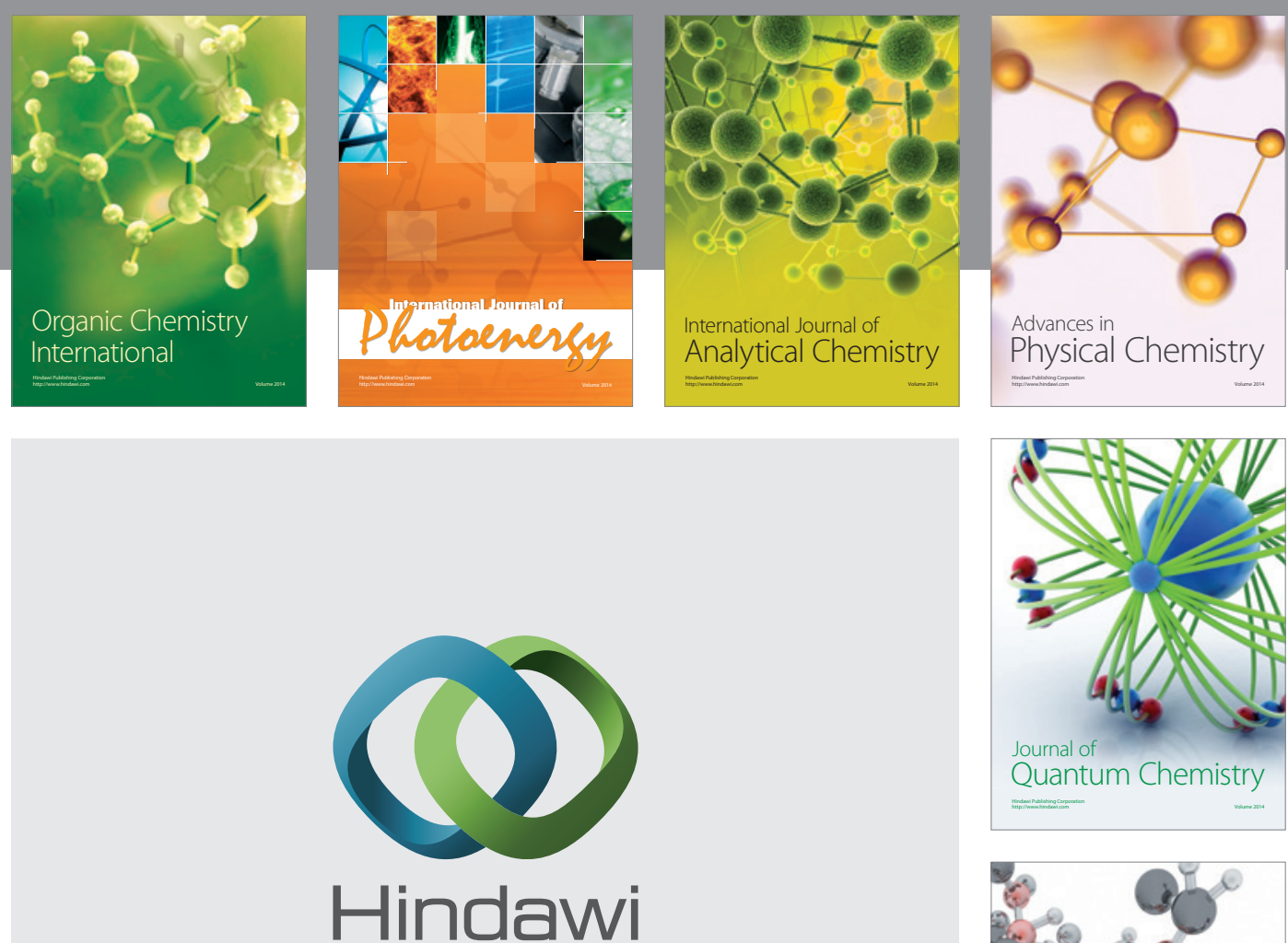

Submit your manuscripts at

http://www.hindawi.com

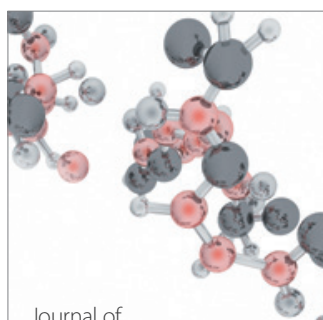

Analytical Methods

in Chemistry

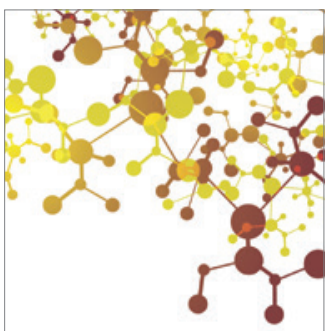

Journal of

Applied Chemistry

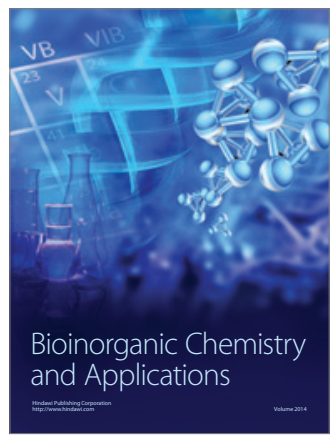

Inorganic Chemistry
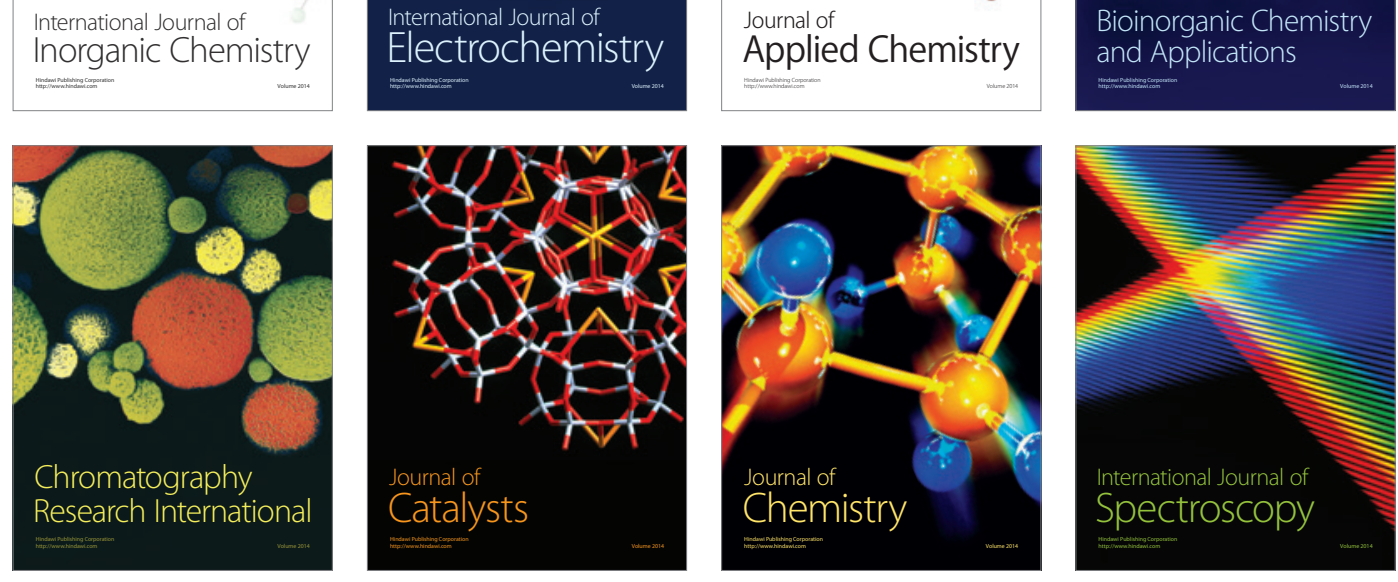